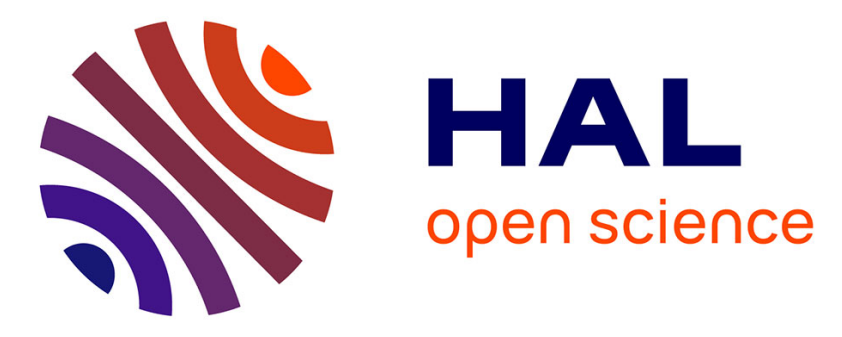

\title{
Collaborative Pseudo-Haptics: Two-User Stiffness Discrimination Based on Visual Feedback
}

Ferran Argelaguet Sanz, Takuya Sato, Thierry Duval, Yoshifumi Kitamura, Anatole Lécuyer

\section{- To cite this version:}

Ferran Argelaguet Sanz, Takuya Sato, Thierry Duval, Yoshifumi Kitamura, Anatole Lécuyer. Collaborative Pseudo-Haptics: Two-User Stiffness Discrimination Based on Visual Feedback. Eurohaptics 2014, 2014, Versailles, France. pp.49 - 54, 10.1007/978-3-662-44196-1_7 . hal-01086248

\section{HAL Id: hal-01086248 https://hal.inria.fr/hal-01086248}

Submitted on 23 Nov 2014

HAL is a multi-disciplinary open access archive for the deposit and dissemination of scientific research documents, whether they are published or not. The documents may come from teaching and research institutions in France or abroad, or from public or private research centers.
L'archive ouverte pluridisciplinaire HAL, est destinée au dépôt et à la diffusion de documents scientifiques de niveau recherche, publiés ou non, émanant des établissements d'enseignement et de recherche français ou étrangers, des laboratoires publics ou privés. 


\title{
Collaborative Pseudo-Haptics: Two-User Stiffness Discrimination Based on Visual Feedback
}

\author{
Ferran Argelaguet, Takuya Sato, Thierry Duval, \\ Yoshifumi Kitamura, and Anatole Lécuyer \\ Tohoku University Research Institute of Electrical Communication \\ Inria Rennes \\ Université de Rennes 1, IRISA \\ \{takuyas6, kitamura\}@riec.tohoku.ac.jp, \\ \{fernando.argelaguet_sanz, anatole.lecuyer\}@inria.fr, \\ \{thierry.duval\}@irisa.fr
}

\begin{abstract}
Pseudo-Haptic feedback has been the object of several studies exploring how haptic illusions can be generated when interacting with virtual environments using visual feedback. In this work we explore how the concept of pseudo-haptic feedback can be introduced in a collaborative scenario. A remote collaborative scenario in which two users interact with a deformable object is presented. Each user, through touch-based input, is able to interact with a deformable virtual object displayed in a standard display screen. The visual deformation of the virtual object is driven by a pseudo-haptic approach taking into account both the user input and the simulated physical properties. Particularly, we investigated stiffness perception. In order to validate our approach, we tested our system in a single and two-user configuration. The results showed that users were able to discriminate the stiffness of the virtual object in both conditions with a comparable performance. Thus, pseudo-haptic feedback seems a promising tool for providing multiple users with physical information related to other users' interactions.
\end{abstract}

Keywords: Pseudo-haptics, User Interfaces, Multi-user Interaction

\section{Introduction}

Collaborative interaction has a wide range of applications such as virtual prototyping, training environments, project reviews and video games. In such applications, it is critical to ensure the communication between users (user awareness). All users must have knowledge about the state of the system and the actions performed by other users. In this work we explore how the concept of pseudohaptic feedback can be introduced in such multi-user scenarios. Specifically, how the perception of physical properties of objects can be enhanced during of coexploration and co-manipulation. 
Pseudo-haptic research has been conducted in order to explore how physical properties of virtual objects (e.g. stiffness, friction, mass) can be simulated without the need of dedicated haptic devices [5]. By delivering appropriate visual feedback accounting for users' actions and the simulated physical properties, some kind of "haptic illusions" can be induced [6]. Among them, the first pseudohaptic paper focused on stiffness perception [5]. Users, by interacting with an isometric input device manipulated a virtual piston displayed on a computer string. The user could displace the virtual piston according to simulated stiffness. Varying the degrees of visual compression of the virtual piston leaded to different levels of perceived stiffness. However, pseudo-haptic studies have not only considered the simulation of physical parameter (e.g. stiffness, torque, friction). For example, Lécuyer et al. [4] explored how the relief of textures could be simulated by adjusting the Control/Display (CD) ratio of the mouse cursor based on the underlying texture information. Other studies have focused on the perception of the shape of 3D objects [1] or on graphical user interfaces [7].

In this research, in contrast to existing pseudo-haptic research on stiffness perception [5], we investigate the stiffness perception when two users interact in a non-colocated setup with deformable objects. The proposed setup enables two users to interact through a tactile interface with a deformable object. The feedback provided to the user is only visual, which is expected to enable stiffness discrimination [2]. The interaction with the deformable object is dependent on the users' actions and its physical properties. The results showed that participants were able to perceive the stiffness of virtual objects during co-manipulation through pseudo-haptic feedback. From this, we conclude that pseudo-haptic feedback can be introduced in collaborative environments. The remaining of the paper details the interaction model and its evaluation.

\section{Concept of Pseudo-Haptic Stiffness Simulation in a Two-User Collaborative Scenario}

The virtual environment considered is composed by a deformable object (cube), and two actuators at each side (see Figure 1 left). In our two-user scheme, each user is able to manipulate one actuator in order to interact with the deformable object. For simplicity, the actuators only have one degree of freedom (horizontal axis). The system is designed in a way that users, in order to deform the object, have to work in a coordinate way. For example, if only one user is pushing, it results only in the translation of the cube without any deformation. In such a system, we have to determine (1) how the user input determines the force exerted by the actuator, and (2) how the forces applied by both actuators modify the state of the deformable object.

Regarding the user input, in our prototype, each user interacts with one of the actuators by moving his finger along a touch surface (see Figure 1 right). The force $(F)$ delivered by the actuator is linked with the amount of displacement accumulated is $F=\Delta x_{f} / a$. Where $\Delta x_{f}$ is the displacement of the user's finger in the touch surface and $a$ is a scaling factor. Each actuator is manipulated 
independently. When the user removes the finger from the tactile surface the actuator will no longer exert any force $(F=0)$.

Considering the environment and the user interaction, the system presents five different cases. They are characterized by the force delivered by each actuator (left $F_{l}$ and right $F_{r}$ ) and whether the actuators are in contact with the deformable object or not:

1. The actuator is not in contact with the interactive object. The movement of the actuator is mapped directly with the user's input.

2. Only one actuator is pushing the interactive object. The actuator displaces the object along the horizontal axis with a 1:1 CD ratio.

3. Both actuators are in contact with the interactive object but one of them does not deliver any force. The behavior is the same as case 2 but the actuator with $F=0$ is also displaced.

4. Both actuators are in contact with the interactive object, and $F_{l}>0$ and $F_{r}>0$. In this case, the force is transmitted to the deformable object which is deformed based on Hooke's law $x_{d}=F / k . k$ determines the stiffness of the virtual cube and $x_{d}$ is the compression of the cube in the x-axis. The force applied is $F=\min \left(F_{l}, F_{r}\right)$. The exceeding force will displace the object and the actuator delivering less force.

5. The virtual object is compressed and $F=0$. The virtual object will recover its original size (elastic deformation). If an actuator is in contact with the virtual object and their force is equal to zero, it will be displaced. The recovery time was not dependent on the cube's stiffness.

The simulation requires participants to interact synchronously when interacting with the virtual object. If both users do not deliver a similar force it will result in the displacement of the virtual object without any deformation.
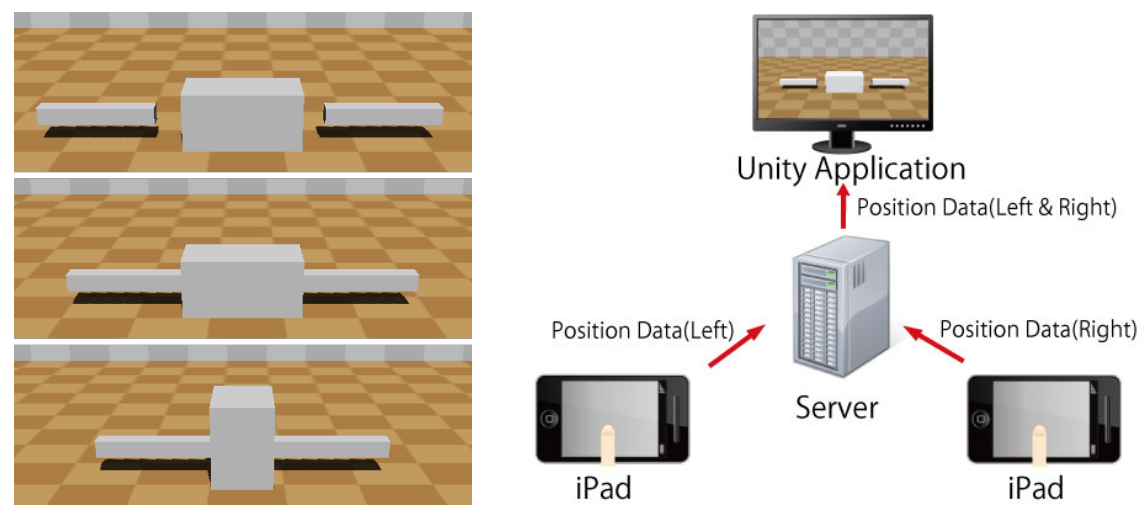

Fig. 1. (Left) the virtual environment considered is composed by one interactive object (center object) and two actuators. The deformation of the virtual object is only triggered when both users interact synchronously. (Right) System's architecture. Each user controls one actuator by swiping his finger on a touch device. 
The pseudo-haptic feedback is linked with the stiffness of the deformable object which modifies the force required to deform it. The higher the value of $k$ is, the higher is the force required to deform the object and vice-versa. Additionally, to increase the visual feedback provided, we preserved the volume of the object while it is being deformed (see Figure 1 left).

Implementation details. Rendering and simulation were handled by a Unity 3D application while the communication between the touch devices (iPads) and the main application was achieved using websockets. The system architecture is displayed in Figure 1.

\section{User Evaluation}

A user evaluation of the system was conducted in order to explore its usability and explore the effects of the user collaboration on the perception of the pseudohaptic feedback. The questions posed in the evaluation were the computation of the Just Noticeable Differences (JND) of the virtual stiffness and whether the fact that both users had to interact synchronously influenced their perception or not. These results are needed both to validate our approach and to provide guidelines to the integration of the proposed effect in a real application.

Design and Procedure. The evaluation followed a 2AFC (two alternative forced choice) procedure with two main conditions: single-user (S) and multi-user (M). For each trial, participants were presented with two virtual cubes (one after another) with different stiffness coefficients $(k)$. Participants had to determine which one was the stiffest. While for the multi-user condition we used the system described in Section 2, for the single-user condition, the user interacted with two iPads at the same time with his two index fingers. For the 2AFC task, the comparisons were based on one reference value $\left(k_{f}=4\right)$ and six comparison values $( \pm 30 \%, \pm 20 \%, \pm 10 \%)$. The order of the conditions (single vs multi) was balanced and the comparisons randomized. For each combination, users performed ten repetitions, resulting in a total of $2 \times 6 \times 10$ (120) trials. Regarding the procedure, for each trial, users were presented with a discrimination task. They had five seconds to interact with each virtual cube and then, they had to answer which cube was the stiffest. Users were able to answer the question through a GUI displayed on their iPads. For each trial, we recorded the answer for the question "Which virtual cube is the stiffest?". Furthermore, we also recorded the displacement of the virtual cube and the difference between the force applied by each effector, which are measures of the users' synchronization. Our hypotheses were [H1]: Stiffness discrimination accuracy will be higher for the single user condition and [H2]: The synchronization between both effectors will be higher for the single-user condition.

Participants. 16 users aged from 21 to 31 (11 male and 5 female) took part in the experiment. All users had no known perception disorders, and used their dominant hand to perform the task. 
Apparatus. The evaluation was conducted using two displays of 20" (see Figure 2 right). Users interacted with the application sitting down, at $50 \mathrm{~cm}$ from the display. In the multi-user condition, users were not able to see neither the others user's display nor the others interface. Oral exchange was not allowed.

Results. The analysis of the results followed a classical psychophysical analysis as described in [3]. First, we consider the answers in which the reference is considered to be stiffer (see Figure 2 left). The JND threshold is computed as the difference between the reference value and the value of the psychophysical curve $\left(f(x)=1 /\left(1+e^{\frac{x-\alpha}{\beta}}\right)\right)$ at the $84 \%$ ordinate. Multi-user: $\Delta I_{M}=4-2.93=1.07$ and single-user: $\Delta I_{S}=4-2.96=1.04$. With this information, we compute the Weber fraction as $k=\Delta I / I$ where $I$ is the value of the reference stiffness. This computation results in $k_{M}=0.267$ and $k_{S}=0.26$.

The two-way ANOVA of Condition (multi,single) and Comparison $( \pm 30 \%$, $\pm 20 \%, \pm 10 \%$ ) versus Accuracy showed a significant difference for Comparison $\left(F_{5,75}=26.12 ; p<0.001\right)$ but no significant difference was found between conditions $\left(F_{1,75}=0.49 ; p=0.494\right)$. The fact that there are no significant differences on the user accuracy is consistent with the fact that the Weber fractions for each condition are similar. In contrast, when analyzing the data regarding the synchronization between both actuators the two-way ANOVA for the cube displacement showed a main effect for Condition $\left(F_{1,15}=712.43 ; p<0.001\right)$. Posthoc tests (Bonferroni) showed that the cube displacement was significantly lower $(p<0.05)$ for the singe-user condition $(38 \%$ smaller $)$. Regarding the force difference, there is also a significant effect on Condition $\left(F_{1,15}=411.93 ; p<0.001\right)$. Post-hoc (Bonferroni) tests showed that the difference was significantly higher $(p<0.05)$ for the multi-user condition $(29 \%$ higher $)$.

Discussion. The results show that users were able to perceive differences of stiffness with a comparable performance for single and multi-user conditions (similar Weber fractions and no significant differences in accuracy). Although we expected a reduced discrimination for the multi-user condition, this was not
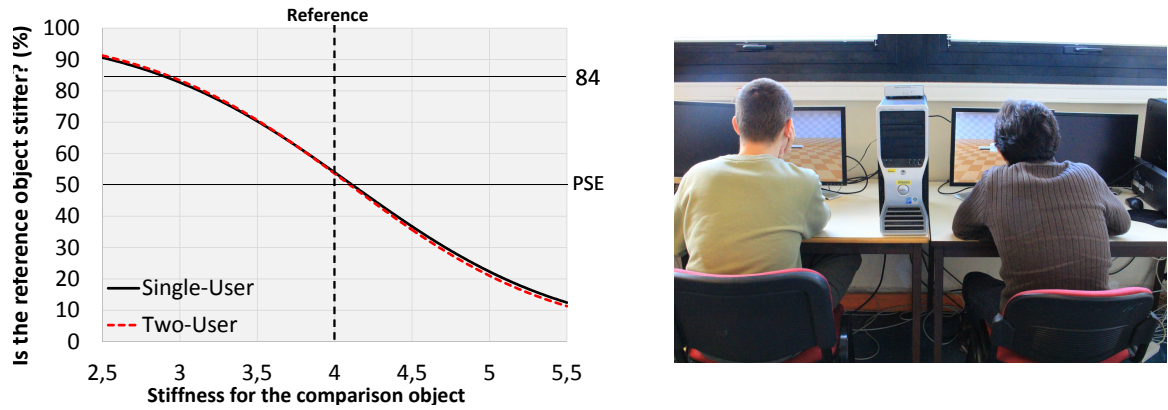

Fig. 2. Left, psychometric curves obtained for the single-user $(\alpha=4.113, \beta=0.711)$ and multi-user $(\alpha=4.098, \beta=0.681)$ conditions. Right, experimental setup. 
the case, thus we reject [H1]. Nevertheless, considering the results on cube displacement and force difference, we clearly observe differences in the degree of synchronization between conditions. Both measures are correlated, the higher is the difference between forces the more the cube is displaced. The deformation is determined by the minimum of the forces delivered by the effectors. In both cases, the values were higher for the multi-user condition, thus we accept [H2]. Hence, for the given simulation, users seem to perceive the stiffness property of the deformable object at the same level between single and multi-user conditions, although their motions are naturally less accurate during a co-manipulation.

\section{Conclusion}

In this work, we have studied the introduction of pseudo-haptic feedback in a collaborative perceptive task: two-user stiffness discrimination task based on visual feedback and tactile input. We have proposed and evaluated the interaction model. In the proposed simulation, two users could feel the stiffness of a deformable cube in a collaborative task. The evaluation conducted investigated the effects of the collaborative interaction in terms of user perception and synchronization. Although we expected a decrease in performance of stiffness discrimination for the collaborative task, the results surprisingly showed that it was not the case. The Weber fraction for single-user was 0.267 and 0.26 for the multi-user scenario. This result could be applied in various applications such as future remote co-exploration, assembly/maintenance simulations or multi-player video games.

\section{References}

1. Y. Ban, T. Kajinami, T. Narumi, T. Tanikawa, and M. Hirose. Modifying an identified curved surface shape using pseudo-haptic effect. In IEEE Haptics Symposium, pages 211-216, Mar. 2012.

2. K. Drewing, A. Ramisch, and F. Bayer. Haptic, visual and visuo-haptic softness judgments for objects with deformable surfaces. In World Haptics, pages 640-645. IEEE, 2009.

3. G. A. Gescheider. Psychophysics : Method, Theory, and Application. Lawrence Erlbaum Associates, New Jersey, US, 1985.

4. A. Lécuyer, J.-M. Burkhardt, and L. Etienne. Feeling bumps and holes without a haptic interface. In Proceedings of the 2004 conference on Human factors in computing systems - CHI '04, pages 239-246, New York, New York, USA, 2004.

5. A. Lecuyer, S. Coquillart, A. Kheddar, P. Richard, and P. Coiffet. Pseudo-haptic feedback: can isometric input devices simulate force feedback? In IEEE Virtual Reality, pages 83-90, 2000.

6. A. Pusch and A. Lécuyer. Pseudo-haptics : From the Theoretical Foundationsto Practical System Design Guidelines. In 13th international conference on multimodal interfaces, page 57, 2011.

7. M. E. Rodgers, M. E. Rodgers, R. L. Mandryk, and K. M. Inkpen. Smart sticky widgets: Pseudo-haptic enhancements for multi-monitor displays. In SmartGraphics, pages $194-205,2006$. 